\title{
Fluctuations importance and control in biological systems
}

\author{
— Bahram Houchmandzadeh and Irina Mihalcescu - DOI: 10.1051/epn/2011606 \\ Université Grenoble 1 / Centre National de la Recherche Scientifique, \\ - Laboratoire Interdisciplinaire de Physique, Unité Mixte de Recherche 5588, Grenoble, F-38041, France
}

Living cells are miniature chemical 'factories'. Unlike their macroscopic counterparts, due to the small number of molecules involved, the reactions that take place are not fully deterministic. 'Probability' management is part and parcel of cell life and leaves its mark at all length scales of living matter. We review some aspects of 'stochastic noise' management, which has profoundly changed our vision of living systems during the past ten years.

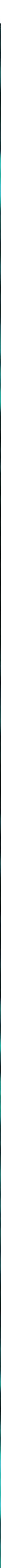


A deterministic machine will always give the same output in response to a given input, while a probabilistic machine will give different outputs more or less widely distributed around a mean value (Figure1). The standard deviation of the output values divided by their mean is what we call the noise amplitude in this article. If this noise is small, the machine is considered deterministic. This is the case for macroscopic chemical reactions, and the usual differential equations of chemical kinetics are sufficient to describe the evolution of concentrations.

When the number of molecules is small, fluctuations are large and the mean value of the molecules number is not a good description of the reaction. Often, in bacteria, chemical reactions are controlled by very low numbers of proteins (Figure 1). A typical example is the bacterium Escherichia Coli (a host of our intestine): $80 \%$ of its genes produce proteins with less than 100 copies/cellular cycle. The noise amplitude for these proteins is in the range of $5 \%$ to $65 \%$. Looking at a col- lection of bacteria, we have to expect that the same reaction will have different outcome in similar individuals (see an example in the introductory picture).

One of the first experiments to show a "non genetic individuality" was performed by Novick and Weiner in 1957, when none of the fine molecular tools of today were available and scientists had a very crude notion of what was going on inside a cell. By observing a population of E. Coli, genetically identical and in the same bath, they showed two distinct behaviours: some were able to digest the sugar lactose, and some were not. Even more troubling: this behaviour, related to the presence of the enzyme $\beta$ galactosidase, was inherited by the offspring of each bacterium. We now know that the concentration of this enzyme, initially under DNA control, can have two stable states of concentration. This bistability is due to the presence of regulatory positive feedback loops. The bacterial chemical reactor falls into one of the two states by random fluctuations, remains there and the offspring inherits this metabolic state.

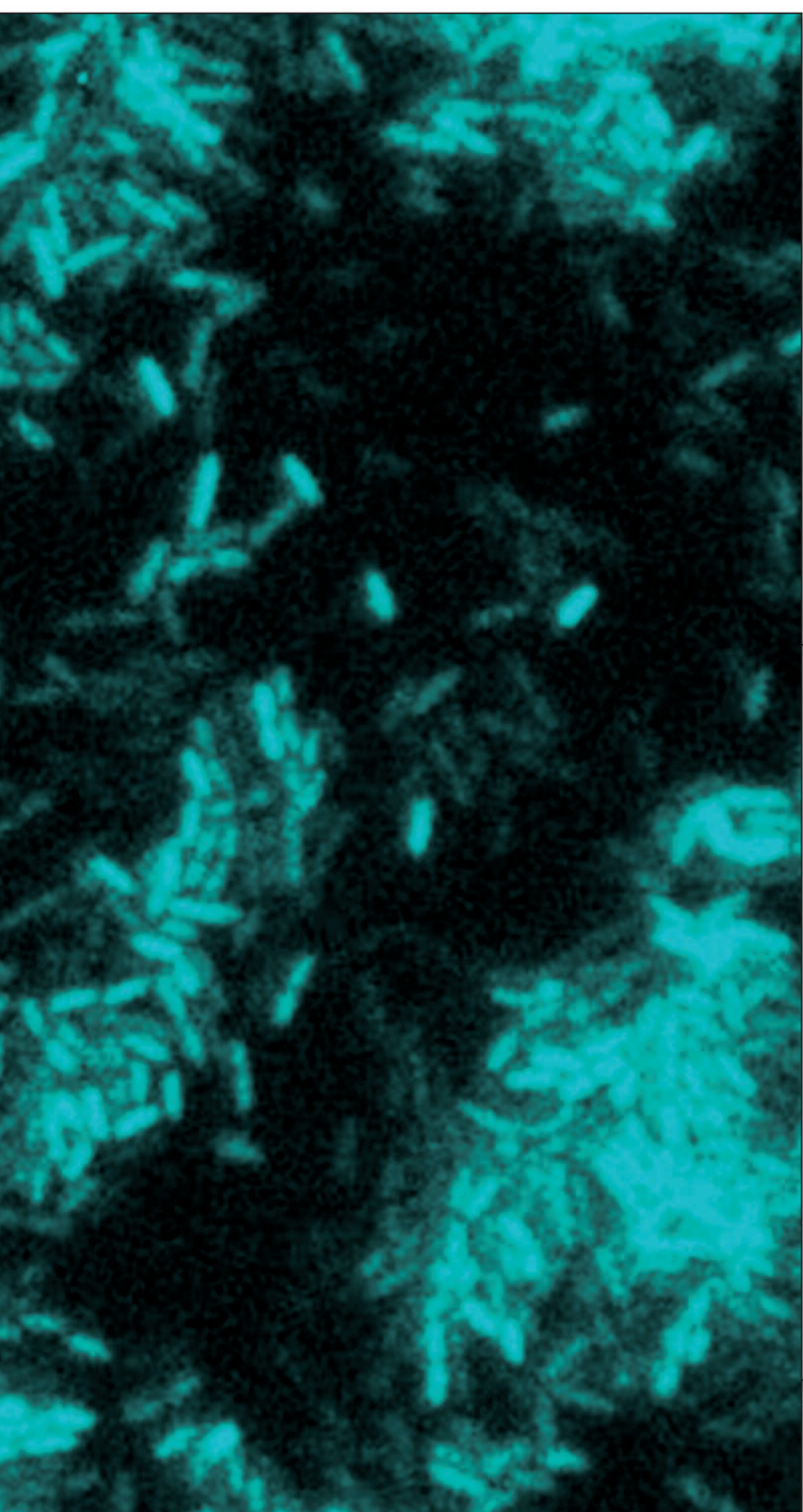

\section{In order to function, an organism has to ensure the fidelity of its response to a given stimulus.}

$\varangle$ Bioluminescence images of rod shaped marine bacteria V. Harveyi (1.5 $\mu \mathrm{m} \times 0.5 \mu \mathrm{m})$. These bacteria are naturally bioluminescent upon addition of an inducer to the medium.

V FIG. 1: Schematic representation of the transcription chemical reaction. Left panel: the probability of observing $n$ molecules produced in a simple chemical reaction at $\approx$ steady state, as a function of the strength of the chemical reaction $k_{+} / k_{\text {, , where }} k_{+}$and $k$ are the forward and backward reaction rate, respectively; the mean number of molecules and the fluctuation amplitude can be read directly on the chart. When $k_{+} / k \approx 1$, the average number of molecules is small and the fluctuations are dominant. Right panel: RNA production from DNA substrate (transcription). $E$ symbolizes both the enzyme RNA-polymerase (Pol) and the DNA substrate (in blue) ; $B$ is for nucleic acids (dNTP, green) ; finally, $A$, the product of the reaction represents the RNA produced (in red).
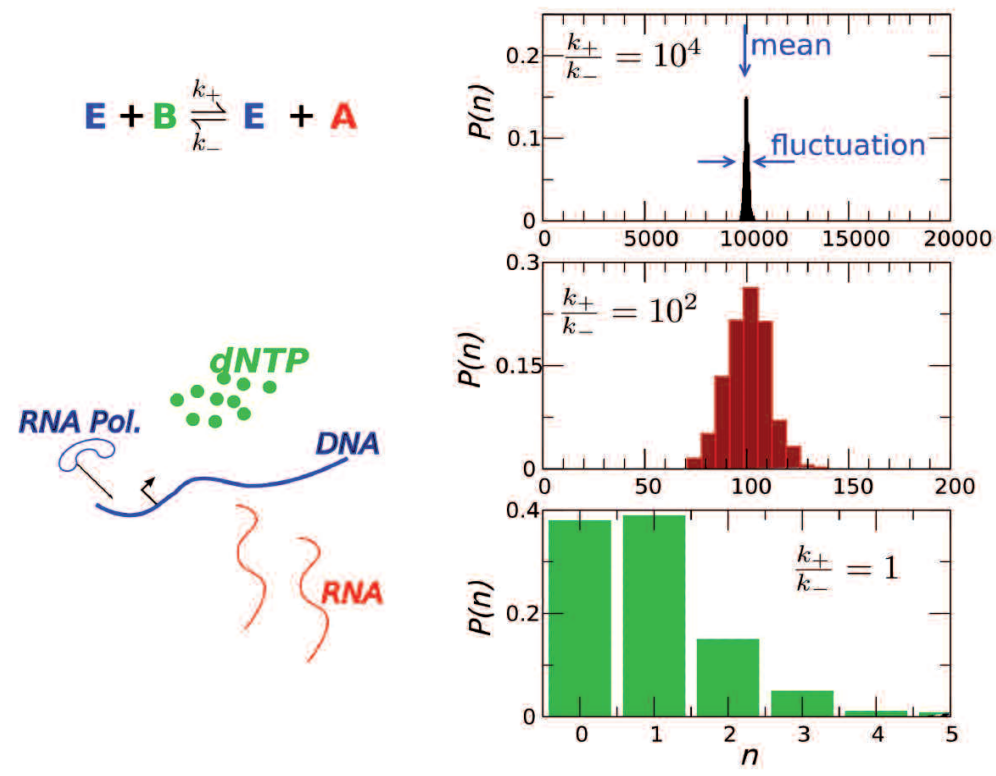
V FIG. 2:

Optical measure-

ment of protein

concentrations.

Left panel: the

gene $x$ encoding

the studied pro-

tein is fused into

the fluorescent

protein gene by

"cut and paste"

molecular

biology tools.

The molecular

machinery will

read this DNA

sequence, then

transcribe it into

messenger

RNA and trans-

late it into new

chimerical

proteins $\mathrm{X}$ which

will contain a

supplementary

fluorescent

domain.

Right panel: Cells

producing this

protein will be

fluorescent and

the level of this

fluorescence is

proportional to

the quantity of

protein inside,

which can be

measured under

a microscope.

Measuring a

few hundred

cells allows

quantifying the

variability of the

cell's response

when producing

the protein $X$.
- The technical advances in molecular biology and the discovery of fluorescent markers brought the subject of fluctuations to the forefront in the 90's. In these techniques, the gene encoding a normal protein is forced to contain also the gene of a fluorescent protein (such as GFP -Green Fluorescent Protein). Whenever the protein is produced, its presence is directly detected in living cells by fluorescence (see Figure 2). It soon became clear that the variability in cell behaviour was not an exception but the rule in a wide variety of systems: from protein production in bacteria and eukaryotic cells (such as our red blood cells) to more complex system such as the oocytes maturation mediated by progesterone, embryonic development, social behaviour. The fluctuations are the combined result of many factors, like the available enzyme in the whole cells (e.g. RNA polymerase in Fig.1) and the characteristic noise of each chemical reaction. Scientists were able to perform clever experiments to distinguish these different sources of noise and propose a general mathematical framework for them [1].

\section{Controlling fluctuations}

In order to function, an organism has to ensure the fidelity of its response to a given stimulus and keep the amplitude of the fluctuations in check. A large set of cell genetic and metabolic regulatory circuits are dedicated to this task and we understand the general principles for the simplest cases. For example, human engineers use negative feedback loops for high fidelity amplification of a signal This mechanism is also widespread in the living world, and bacteria have an important number of genes that are selfregulated like that. When very high precision is needed, one can witness "living" engineering gems of high fidelity. A recent example is the circadian clock in cyanobacteria (a two billion years old organism which generated the rise of the atmospheric oxygen). Widespread in the living world, result of the adaptation to the night/day terrestrial cycle, the circadian clock is a self-sustained biochemical oscillator with an approximately $24 \mathrm{hr}$ period. In cyanobacteria this oscillator has a strong temporal stability: it keeps the phase of oscillation constant during many months [2] in the absence of any outside cue, like light or temperature (see Figure 3). For a long time, it was thought that this precision is due to communication among bacteria, raising the effective number of molecules of the subsequent chemical reactions. We have shown that this is not the case: each

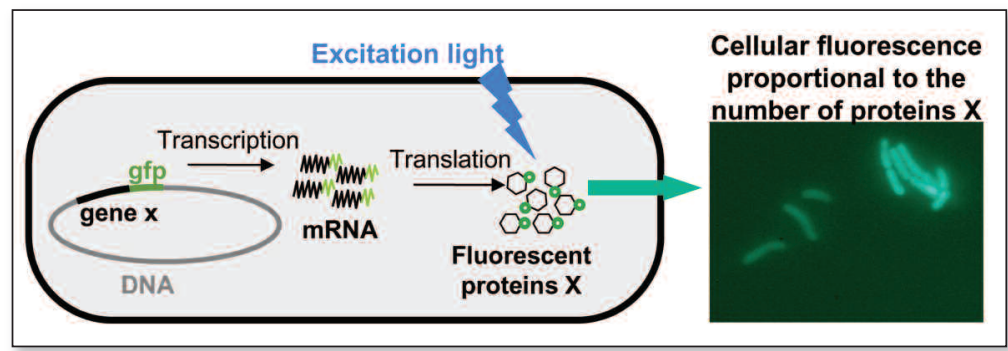

bacterium, using a small number of molecules, is capable of keeping its phase, a fact which seems to defy the rules of thermodynamics [3].

Another example is the embryonic development where different groups of cells progressively specialize from stem cells into tissues. It is vital for an animal to maintain a high precision in the size, and especially in the proportion of different tissues. The generic program used for differentiation is the following. A spatially varying concentration of a signalling molecule is built across the embryo (or part of it). Each cell "reads" this signal, and selects a binary fate according the local concentration of the signal at its position. Each differentiated cell can become itself a producer of a new signalling molecule and so on. This cascade converts a crude initial gradient into a fine genetic positioning system, where each cell, according to its position in the embryo, receives precise instruction to differentiate. This program, however, is too sensitive to noise and unable to ensure proportion fidelity. The generic program has to be completed by many additional correcting circuits, which indeed ensure better fidelity than one-cell fidelity. We can measure the extreme efficiency of these additional circuits, even though we don't completely understand their blue-print [4].

\section{Using Fluctuations}

The living world also exploits random fluctuations. The best known example is of course the Darwinian evolution, which is the founding principle of biology: the very act of duplication of an organism is subject to noise and produces a small proportion of mutants, which can be selected if they are better adapted to the environment. Darwinian evolution, based on the noise at the level of the genome, happens over long time scales. The same mechanism, at the level of gene products, can produce adaptation on short time scales. This was recently brought to the forefront in the bacterial persistence problem of the E. Coli: in a given population of bacterial clones, a small proportion can resist anti bacterial agents for a long time. It was shown by N. Questembert-Balaban that this bacterium has two metabolic states, switching randomly between them. In the favourable state, the bacteria are strong importers of their resources from the outside world and duplicate fast, at the expense of an increased sensitivity to antibacterial agents easily imported inside the cell. In the unfavourable state, the bacteria seldom communicate with the outside world, at the expense of very slow division. The random switch between the two states insures that a small proportion of the population is always maintained in the unfavourable state, and can repopulate the ecological niche after the end of a cataclysmic antibacterial event. In a more general framework, it appears that in fluctuating environment, a gene whose products show some variability can give an advantage to its bearer. 


\section{Fluctuation at large spatial scales}

Fluctuations at the individual level can have consequences at the ecological level and over large spatial scales. One important example is the clustered distribution of species in natural environment: individuals of a given species are not homogeneously distributed in space. This phenomenon was first observed for plankton at ocean's surface. Since then, reviews of more than thousand species in different kingdoms have shown that the clustered distribution is a general rule of ecology. One obvious cause of the distribution heterogeneity is geography itself: lakes, mountains, quantity of available sun... This changes the local carrying capacity and should have an effect on the number of individual inhabiting the place. There is, however, a deeper cause, which is the randomness and asymmetry between birth and death: a new individual always appears close to its parent, but can die everywhere. This asymmetry favours short distances between individuals despite their random movement. We have recently shown that indeed, in a controlled ecological experiment on amoebae, in the absence of any outside clue, the spatial distribution of individuals becomes extremely clustered after a few generations (see Figure 4). In addition all statistical properties of the distribution are predicted by a probabilistic theory taking into account random birth, death and diffusion [5]. The clustered distribution in natural communities therefore should indeed be the rule, and not a source of surprise.

\section{Conclusion}

Stochastic fluctuations have come to the forefront of biology, thanks to active collaborations between on the one hand physicists, who contributed by their culture of statistical physics and the methods used there and, on the other hand, biologists who brought their knowledge of the complexity of the living world and their mastering of molecular biology tools. The cell is no more considered as a deterministic machine, but possesses a non-genetic individuality provided by amplified fluctuations and maintained by multistable chemical reactions.

Much has been achieved in the understanding of these phenomena but much more remains to be understood. We know many specific genetic circuits inside the cell dedicated to noise management, but we lack a general view on their design principles and their fundamental limitations. We are only just beginning the journey into this field.

\section{About the Authors}

Bahram Houchmandzadeh is directeur de recherche at CNRS. He graduated in statistical physics, and first dedicated his interests to out-of-equilibrium physical systems. He then became interested in biological systems, and worked in various areas like the structure of mitotic chromosomes, embryonic development, population structure in ecology and Evolution.

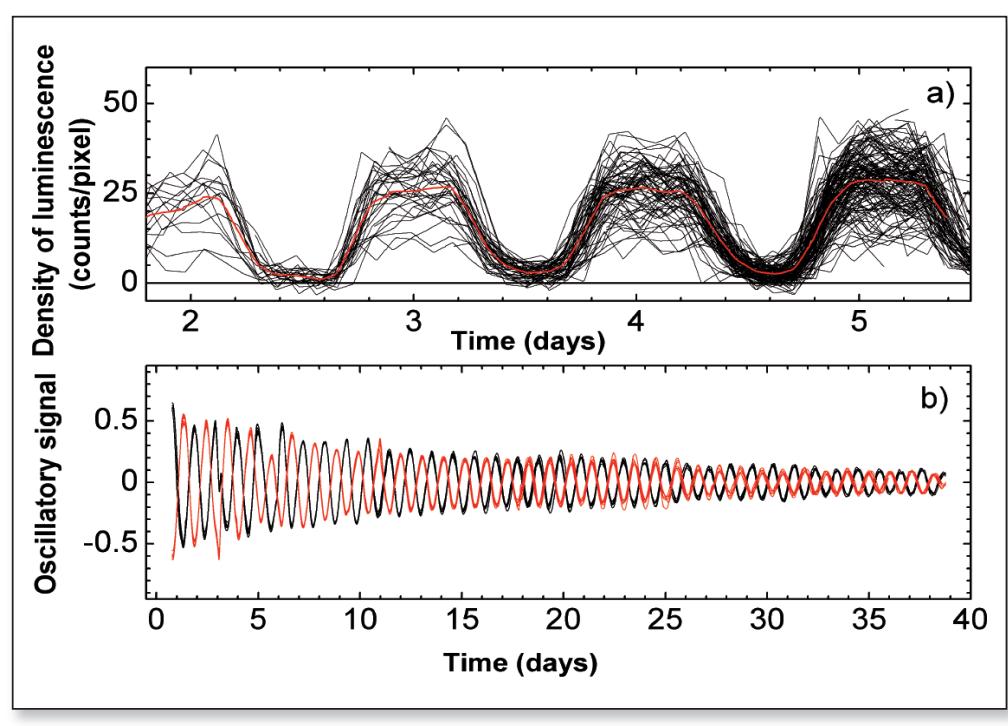

$\triangle$ FIG. 3: Synchronous oscillation of individual bacteria and their progeny. (a) In the cyanobacterium Synechococcus elongatus, the circadian clock adds a periodic component to all protein production. To follow this oscillation we use a bioluminescent reporter. Each black curve represents the signal emitted by one bacterium and the red curve their average. (b) The normalised circadian oscillation of two populations of cyanobacteria (shown in red and black) with opposite initial phases, conserves its phase over many weeks (for each phase 8 to 12 experimental curves are superimposed). Note that only the phase information is relevant as the amplitude is sensitive to metabolic variations along the experiment ([3] and [4])

Irina Mihalcescu is Professor at Grenoble University. Junior member at the Institut Universitaire de France from 2005-2010, she studies the fluctuations in biological systems like the circadian clock, the quorum sensing and the stress response network. She graduated in semiconductor physics and first inquired into the fluorescence mechanisms in porous silicon.

\section{References}

[1] A. Raj, A. van Oudenaarden, Cell 135, 216 (2008).

[2] I. Mihalcescu, W. Hsing, S. Leibler, Nature 430, 81 (2004).

[3] M. Amdaoud, M. Vallade, C. Weiss-Schaber, I. Mihalcescu, Proc. Natl. Acad. Sci. U.S.A. 104, 7051 (2007).

[4] B. Houchmandzadeh, E. Wieschaus, S. Leibler, Nature 415, 798 (2002).

[5] B. Houchmandzadeh, Phys. Rev. Lett. 101, 078103 (2008).

V FIG. 4: The clustered spatial distribution after 9 generations, beginning with a homogeneous initial distribution. Each amoeba ( $D$. Discoidum) has been detected at high magnification and represented here by a dot. Each birth, occurring close to a parent, increases the number of "short" distances. The Brownian motion tends to dilute short distances, but cannot overcome "short distance" creations at two dimensions [5].

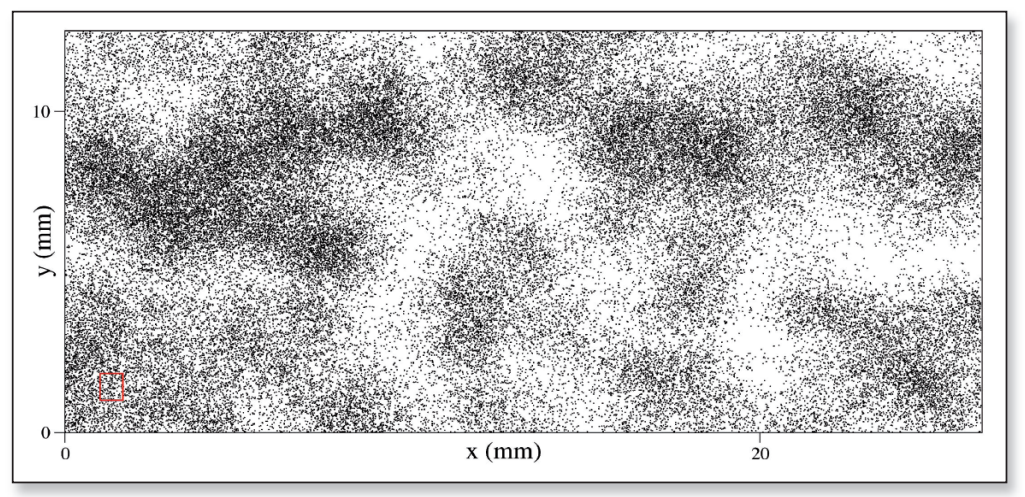

